\title{
Evaluation of Bone-Implant Interface Stress and Strain Using Heterogeneous Mandibular Bone Properties Based on Different Empirical Correlations
}

\author{
Mostafa Omran Hussein ${ }^{1, \odot ~}$ Mohammed Suliman Alruthea ${ }^{1}$ \\ ${ }^{1}$ Department of Prosthodontic Sciences, College of Dentistry in \\ Address for correspondence Mostafa Omran Hussein, BDS, MS, \\ Ar Rass, Qassim University, El-Qassim, Saudi Arabia \\ PhD, Department of Prosthodontic Sciences, College of Dentistry \\ in Ar Rass, Qassim University, El-Qassim 58876, Saudi Arabia \\ (e-mail:m.omran@qu.edu.sa).
}

Eur J Dent 2021;15:454-462

\begin{abstract}
Objectives The purpose of this study was to compare methods used for calculating heterogeneous patient-specific bone properties used in finite element analysis (FEA), in the field of implant dentistry, with the method based on homogenous bone properties.

Materials and Methods In this study, three-dimensional (3D) computed tomography data of an edentulous patient were processed to create a finite element model, and five identical 3D implant models were created and distributed throughout the dental arch. Based on the calculation methods used for bone material assignment, four groups-groups I to IV-were defined. Groups I to III relied on heterogeneous bone property assignment based on different equations, whereas group IV relied on homogenous bone properties. Finally, $150 \mathrm{~N}$ vertical and 60-degree-inclined forces were applied at the top of the implant abutments to calculate the von Mises stress and strain.

Keywords

- CBCT-based finite element analysis

- mandibular bone implant

- heterogeneous bone properties

- computed topography

- bone-implant interface

Results Groups I and II presented the highest stress and strain values, respectively. Based on the implant location, differences were observed between the stress values of group I, II, and III compared with group IV; however, no clear order was noted. Accordingly, variable von Mises stress and strain reactions at the bone-implant interface were observed among the heterogeneous bone property groups when compared with the homogenous property group results at the same implant positions.

Conclusion Although the use of heterogeneous bone properties as material assignments in FEA studies seem promising for patient-specific analysis, the variations between their results raise doubts about their reliability. The results were influenced by implants' locations leading to misleading clinical simulations.
\end{abstract}

\section{Introduction}

Finite element analysis (FEA) is an efficient computational tool that has been used since many years in dentistry (particularly in implant dentistry) to study different implant

published online

January 28, 2021
DOI https://doi.org/

10.1055/s-0040-1721549

ISSN $1305-7456$. treatment designs and materials. To validate FEA and enhance its clinical implications, validation studies are considered supplementary to this technique. Validation can be performed using direct or indirect methods. Direct methods can be based on experimental models, ${ }^{1-3}$ animal trials, ${ }^{4,5}$ human (c) 2021. European Journal of Dentistry.

This is an open access article published by Thieme under the terms of the Creative Commons Attribution-NonDerivative-NonCommercial-License, permitting copying and reproduction so long as the original work is given appropriate credit. Contents may not be used for commercial purposes, or adapted, remixed, transformed or built upon. (https://creativecommons.org/licenses/by-nc-nd/4.0/)

Thieme Medical and Scientific Publishers Pvt. Ltd. A-12, 2nd Floor, Sector 2, Noida-201301 UP, India 
clinical trials, ${ }^{6,7}$ stress analysis methods, ${ }^{8}$ or another FEA software. ${ }^{9,10}$ Conversely, indirect methods include the review of previous studies that address a similar problem to that under investigation. ${ }^{11,12}$ In a literature review, Chang et a ${ }^{13}$ presented a validation level hierarchy based on the evidence required to achieve a satisfactory clinical significance. Recent FEA studies have devoted significant efforts to achieve innovative models with high simulation characteristics, which are able to mimic the clinical environment and thus minimize the need for a validation process. Those studies simulated masticatory muscle traction, ${ }^{14}$ dynamic masticatory forces, ${ }^{15}$ and nonlinear FEA body contacts. ${ }^{16,17}$ In addition, the analysis was enhanced by using anisotropic bone properties. ${ }^{18,19}$

Mandibular bone is an example of the complex heterogeneous structure of human bones; it is mostly composed of cortical and trabecular bone. During the study of biomechanical conditions, particularly in implant dentistry, the mandible has been treated as homogenous tissue with two material properties representing cortical and cancellous bone..$^{20-22}$ In more advanced researches, bone was considered an oblique isotropic or even an anisotropic material to which different material properties were assigned according to the load direction in the $x, y$, and $z$ axes. Although using anisotropic Young's modulus material property is considered relatively accurate, extensive computer resources are needed and, more importantly, the results are not patient specific..$^{19,23}$ Consequently, some researchers adopted patient-specific material assignment. They relied on the correlation between the density of the bony tissues and its elastic modulus; moreover, they considered the correlation between bone density and the patient's gray scale data from a computed tomography (CT). ${ }^{24-}$ ${ }^{30}$ However, it was difficult to confirm whether these correlations were reliable calculation methods. Many techniques have been used to measure bone density and elastic modulus and thus reveal their correlation, such as mechanical testing, ${ }^{27}$ ultrasonic measurement, ${ }^{25}$ and nanoindentation. ${ }^{24}$

Advances in computer software facilitate the use of Hounsfield unit (HU) data from the patient's CT images as an approach for calculating the density and afterwards the elastic modulus of bone. . $431-35^{3}$ These software products are also able to transfer this information to the generated volume mesh, which can be then exported to finite element software for performing the analysis. MIMICS medical software has the unique feature of using customized equations between the $\mathrm{HU}$ and bone density and also between the bone density and elastic modulus. ${ }^{24,36,37}$ Accordingly, the differences between assigning heterogeneous and homogenous bone properties to tibia bone have been assessed using customized equations within FEA and compared with the corresponding experimental values of a long control bone.

The current study hypothesized that conventional calculation methods for creating heterogeneous patient-specific mechanical bone properties do not achieve reliable results when used in implant dentistry FEA, particularly when compared with homogenous material property assignment results. Many of these heterogeneous property methods depend on different equations according to the calculated correlations, which may result in different outcomes and significant inaccuracies. After an intensive literature review, no articles discussing the reliability of using these material property assignment methods for mandibular bone and their effects on the overall results were found; accordingly, the purpose of the study was to compare methods used for calculating heterogeneous patient-specific bone properties with the method based on homogenous bone properties. To this end, the von Mises stress and strain at the peri-implant bone tissue were assessed under vertical and inclined loading conditions. In the present study, the influence of different material assignments and their reliability within the FE model were investigated. A three-dimensional (3D) mandibular model was created, from CT data, followed by adding five 3D implant models. Afterwards, three different correlation equations, between CT gray scale, density, and elastic modulus, were applied to the FE model to form heterogeneous material models. Another FE model based on homogenous material was created and compared with the heterogeneous models. FEA was performed to calculate von Mises stress and strain as postprocessing parameters in both vertical and inclined forces scenarios. The results were studied on each implant site and interpreted quantitatively referencing the homogenous group and qualitatively by the color-coded maps.

\section{Materials and Methods}

After ensuring that the research procedures have been performed in accordance with the Code of Ethics of the World Medical Association (Declaration of Helsinki) for experiments involving humans, the research project was approved from the scientific research ethical committee at ArRass Dental College, Qassim University (local ethical committee, No: A-115). CT images of a fully edentulous female patient, in the Digital Imaging and Communications in Medicine (DICOM) format, were selected from the database of the Oral and Maxillofacial Radiology Centre at ArRass Dental College, Qassim University. The patient was contacted to get her agreement and sign the patient's consent, keeping human subjects' privacy rights. The CT field of view was targeted at the mandibular bone extending to the ascending ramus. The DICOM images were imported to the MIMICS medical software (version 21; Materialise NV, Leuven, Belgium) to create an image mask based on the bone threshold. The mask was smoothed, modified, refined, and then converted into a 3D model of the mandibular bone ( $\boldsymbol{- F i g . 1 )}$. The 3D model was exported to the 3-matic medical software (version 13; Materialise NV).

A $4 \times 12 \mathrm{~mm}$ (diameter $\times$ length) 3D model of an implant with attached abutment was designed in 3-matic. Then, four implant duplicates were created, and the five obtained implants were placed at different positions along the mandibular bone: one at the symphyseal zone, two at the parasymphyseal zone, and two at the posterior zone. The surface meshes of both the mandibular bone and the implant were treated using semiautomatic fixing tools. The surface meshes were then remeshed by adaptive remesh and quality preserving tools to create surface meshes with triangle edge ranging from 0.3 to $1.5 \mathrm{~mm}$. Special refinement was performed at the 


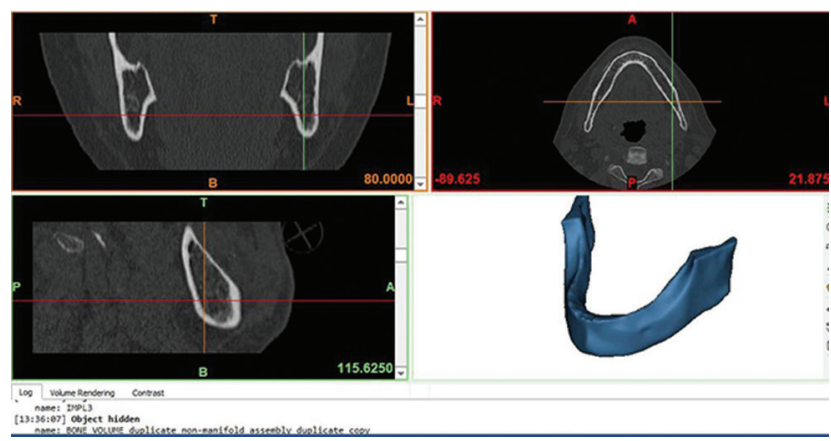

Fig. 1 Creation of the three-dimensional (3D) mandibular bone model in MIMICS software using the assigned mask.
A
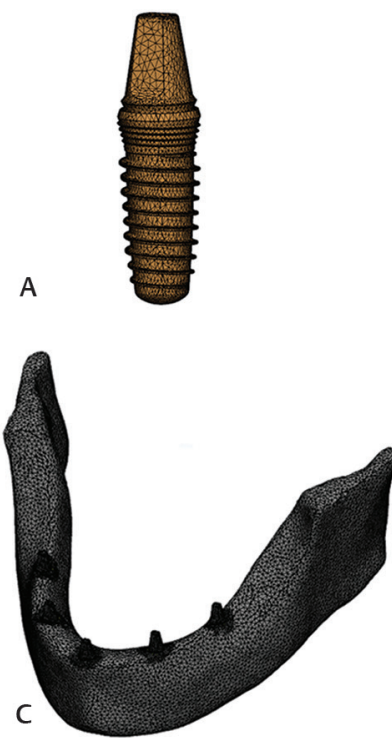

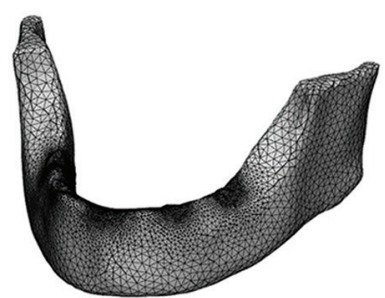

B

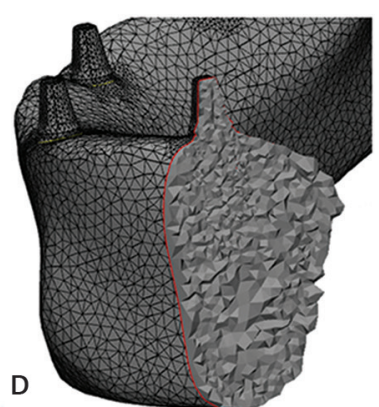

Fig. 2 (A) Implant and abutment designed and meshed in 3-matic software. (B) The bone model was remeshed and refined at the implant intersection. (C) Full and (D) cross-sectional views of the volume mesh for the entire assembly.

small fine areas, such as implant threads and highly curved bony areas, and at the implant-bone intersection areas. A nonmanifold assembly was created including all the models of the study to obtain uniform interfaces throughout the mandibular bone and designed implant. Finally, a volume mesh with a suitable element size (TET-4) was created. A $20 \%$ growth rate, local volume mesh parameter, was considered at implants zones for mesh refinement. The mesh quality was examined for validity to pass the finite element mesh criteria of ANSYS software (ANSYS Workbench version 14; ANSYS Inc., Canonsburg, Pennsylvania, United States). The obtained models and meshes are shown in - Fig. 2.

The volume mesh was reimported to MIMICS for the assignment of different materials. The homogenous implant material properties assigned consisted of single values of density $(\rho)$, Young's modulus $(E)$, and Poisson's ratio, regardless of the grayscale levels in the CT images. The values of density, Young's modulus, and Poisson's ratio used were $4.5 \mathrm{~g} / \mathrm{cm}^{3}, 110$ $000 \mathrm{MPa}$, and 0.3 , respectively. ${ }^{23}$ By contrast, the mandibular bone material properties were assigned based on the CT image grayscale levels, which were used as patient-specific heterogeneous material properties. Therefore, four MIMICS files were created according to the four studied groups with different assigned mandibular bone empirical expressions. The groups were established as follows.

\section{Group I}

The material properties assigned to this group were based on the correlation between the HU and the bone density ${ }^{31}$ :

$$
\rho=114+0.916 \times \mathrm{HU},
$$

while the relation between the bone density and Young's modulus was

$$
\mathrm{E}=0.51 \times \rho^{1.37} .
$$

\section{Group II}

In this group, the relation between the bone density and $\mathrm{HU}$ was calculated from the following equation ${ }^{29}$ :

$$
\rho=0.6618 \times \mathrm{HU}+9.84,
$$

while three different equations were used to calculate the relation between the bone density and Young's modulus, according to the bone density range ${ }^{30}$ ( - Table $\mathbf{1}$ ).

\section{Group III}

The bone density equation used in this group was based on the gray value (GV) rather than on the HU unit. This conversion was done automatically by changing the software setting from $\mathrm{HU}$ to $\mathrm{GV}$ before calculating material data. Accordingly, the following equations were employed ${ }^{24}$ :

$$
\begin{aligned}
& \rho=-13.4+1017 \times \mathrm{GV}, \\
& \mathrm{E}=-388.8+5925 \times \rho .
\end{aligned}
$$

\section{Group IV}

This group represents the conventional group; single values of density and Young's modulus were used for the cortical bone and trabecular bone, and the HU value was used to distinguish between the former $(\mathrm{HU} \rho 1,000)$ and the latter $(\mathrm{HU}>1,000) .{ }^{38}$ The matcne were $\rho=1.3 \mathrm{~g} / \mathrm{cm}^{3}$ and $E=13,700$ MPa, whereas those for the trabecular bone were $\rho=1.1 \mathrm{~g} / \mathrm{cm}^{3}$ and $E=1,370 \mathrm{MPa}^{23}$

The fixed Poisson's ratio value used for all groups was 0.3 . All the generated density values below zero were replaced with the smallest positive density value from the same group. After material property assignment, a color map presenting the material properties with their corresponding number of

Table 1 Relations between bone density and Young's modulus for different bone density ranges ${ }^{29}$

\begin{tabular}{|l|l|l|}
\hline Bone density $\left(\mathbf{g} / \mathbf{c m}^{3}\right)$ & \multicolumn{2}{|c|}{ Young's modulus (MPa) } \\
\hline $0<\rho \leq 0.27$ & $E=33900 \times \rho^{2.20}$ & $(4)$ \\
\hline $0.27<\rho<0.6$ & $E=5307 \times \rho+469$ & $(5)$ \\
\hline $0.6 \leq \rho$ & $E=10200 \times \rho^{2.01}$ & $(6)$ \\
\hline
\end{tabular}



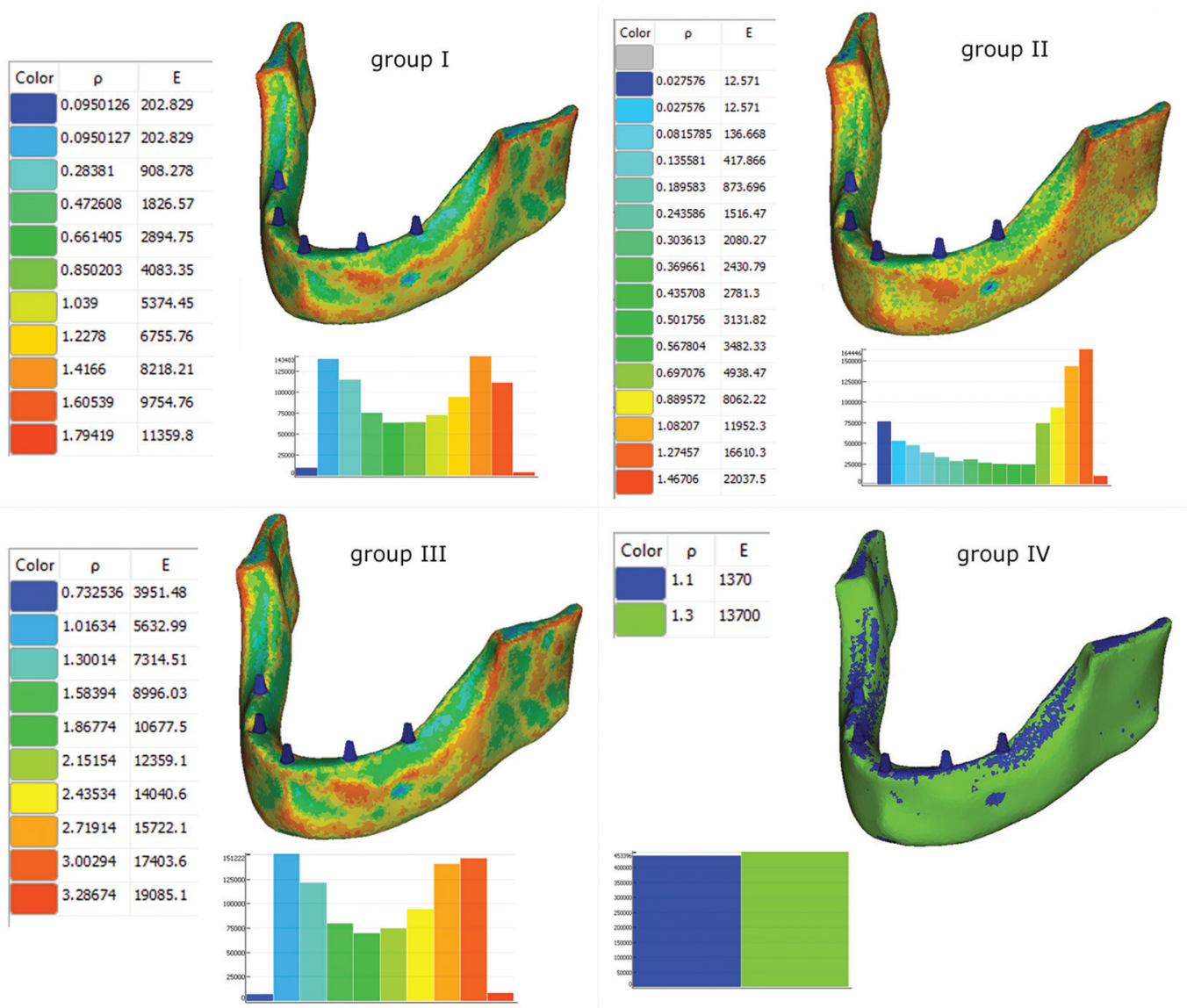

Fig. 3 Color map of correlated elastic modulus and density values on the mandibular model of each group and histograms of the number of elements adopting these values.

assigned elements and values on the 3D model was obtained, as seen in $\boldsymbol{- F i g .} \mathbf{3}$.

Finally, all models were exported as an ANSYS file and a material data file. These files were imported into the finite element modeler module of ANSYS, where the material data files were added as a command file containing the engineering data. Two boundary condition scenarios were considered. The first one assumed a vertical nodal force of $150 \mathrm{~N}$ on the top of each abutment, while the second one assumed a $150 \mathrm{~N}$ force with a 60-degree inclination from the right side as seen from the frontal view. ${ }^{23}$ Constrains were applied on the inner and outer surfaces of the ramus to represent the insertion of the medial pterygoid and masseter muscles, respectively. All contact types were set to "bonded" to prevent node sliding at the interface ( $\boldsymbol{- \text { Fig. }}$ 4). The outcome parameters were the von Mises stress and strain at the bone-implant interface of each implant for both vertical and inclined force conditions.

\section{Results}

von Mises stress and strain values at the bone-implant interface for different implant positions were collected and tabulated, and their color maps were saved to be interpreted. These two parameters were considered for assessing the performance of different material assignment calculation methods based on the grayscale level values and their correlation with bone density and Young's modulus.

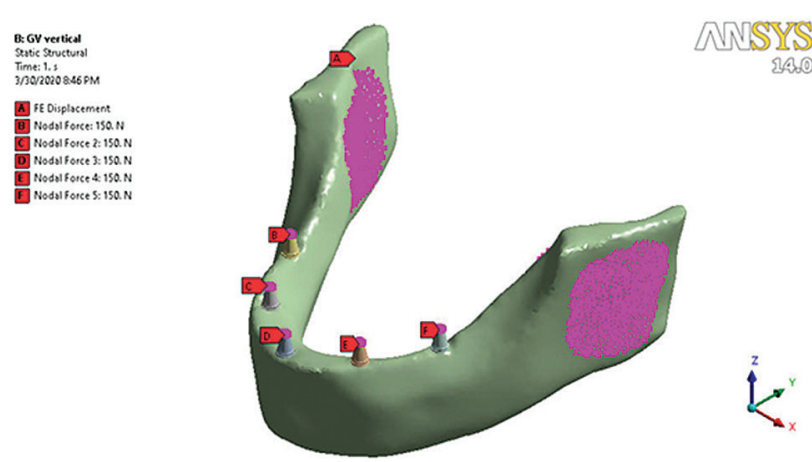

Fig. 4 Boundary conditions applied to the model, including the forces applied on the abutments and the constrains applied on the ramus inner and outer surfaces.

\section{von Mises Stress at the Bone-Implant Interface Upon} Application of a Vertical Force

The change in the generated von Mises stress among the different groups upon vertical force application was affected by the implant position (-Table 2). The bone-implant interfaces surrounding the first and third implants achieved their highest von Mises stress values in group II, followed by group I, then by group IV, and finally by group III. In the second implant, the highest von Mises stress value was observed in group IV, followed by group II, then group I, and finally group III. In the fourth implant, group II showed the highest von Mises 
Table 2 von Mises stresses at the bone-implant interfaces of the different studied groups upon application of vertical (V) and inclined (I) forces (unit: MPa)

\begin{tabular}{|c|c|c|c|c|c|c|c|c|c|c|}
\hline & \multicolumn{2}{|c|}{ Implant 1} & \multicolumn{2}{|c|}{ Implant 2} & \multicolumn{2}{|c|}{ Implant 3} & \multicolumn{2}{|c|}{ Implant 4} & \multicolumn{2}{|c|}{ Implant 5} \\
\hline & $V$ & $I$ & $V$ & $I$ & $V$ & $I$ & $V$ & $I$ & $V$ & $I$ \\
\hline GI & 42.17 & 41.27 & 14.05 & 10.05 & 24.94 & 24.72 & 6.669 & 9.899 & 34.81 & 38.06 \\
\hline GII & 53.47 & 53.27 & 15.84 & 12.75 & 35.78 & 29.20 & 7.882 & 12.76 & 46.04 & 41.47 \\
\hline GIIII & 35.03 & 35.21 & 11.89 & 11.41 & 15.79 & 22.75 & 6.956 & 10.78 & 31.98 & 32.91 \\
\hline GIV & 38.56 & 38.22 & 15.92 & 10.71 & 24.12 & 24.04 & 6.627 & 12.56 & 37.75 & 40.73 \\
\hline
\end{tabular}

Percentage of changes in Von Mises stress compared with group IV for a vertical force

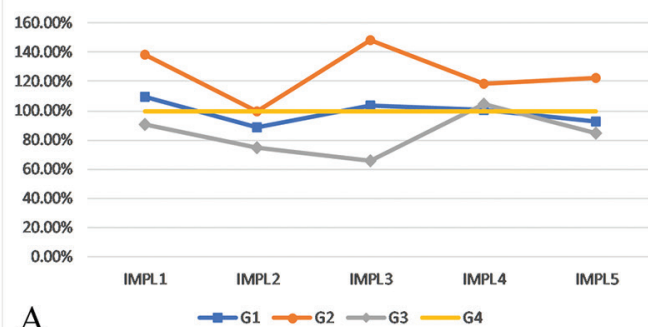

A

Percentage of changes in Von Mises strain compared with group IV for a vertical force

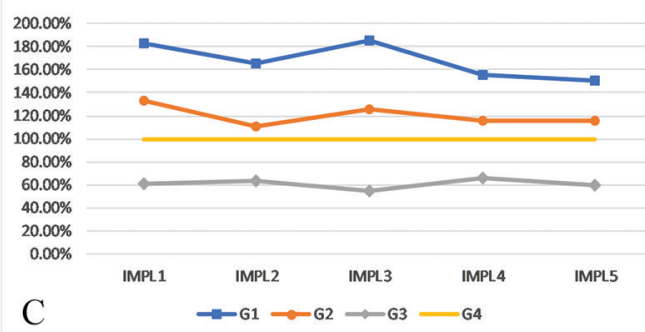

Percentage of changes in Von Mises stress compared with group IV for an inclined force

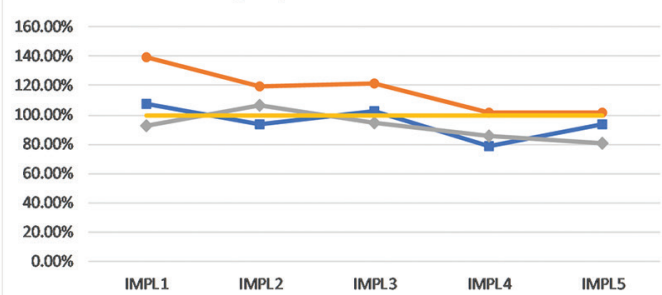

B

Percentage of changes in Von Mises stress compared with group IV for an inclined force

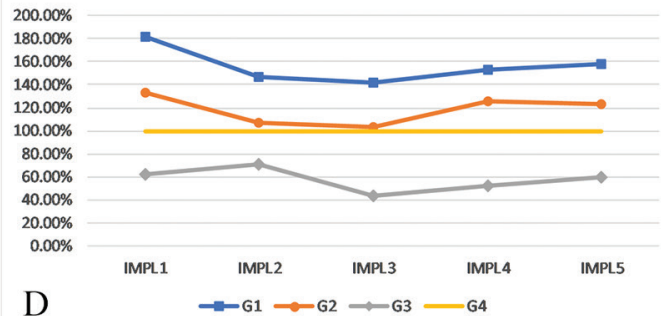

Fig. 5 Percentage of change in the bone-implant interface: von Mises stress of the different groups in comparison with group IV upon the application of a (A) vertical or (B) 60-degree-inclined force. Percentage of change in the bone-implant interface: von Mises strain of the different groups in comparison with group IV upon the application of a (C) vertical or (D) 60-degree-inclined force.

stress value, followed by group III, then by group I, and finally by group IV. A different sequence was observed in the fifth implant: group II presented the highest von Mises stress value, followed by group IV, then by group I, and finally by group III.

In general, upon the application of a vertical force, the von Mises stress results achieved their highest von Mises stress values in group II for all implants, except for implant 2, in which the difference between the highest and second highest values was minimal (0.08 MPa). The lowest von Mises stress values were recorded in group III, except for implant 4 , in which the difference between the lowest and second lowest values was $0.33 \mathrm{MPa}$.

The percentage of change, in von Mises stress values, of the different groups from group IV (i.e., the group with conventional homogenous material properties) was plotted (-Fig. 5). Implant 3 showed the greatest difference among groups I to III, whereas implant 2 showed the smallest difference. Overall, group I was the closest to group IV.

The color maps of the von Mises stress ( - Fig. 6) show higher values in the two distal implants (implants 1 and 5), followed by the midsagittal implant (implant 3). It should be noted that stress concentration is mainly observed at the marginal bone, particularly on the facial side of the boneimplant interface.

\section{von Mises Stress at the Bone-Implant Interface Upon Application of an Inclined Force}

For the case in which an inclined force was applied (-Table 2 ), the highest von Mises stresses generated in the first and third implants were observed in group II, followed by group I, then by group IV, and finally by group III. In the second implant, the highest von Mises stress value was observed in group II, followed by group III, then by group IV, and finally by group I. In the fourth implant, the highest von Mises stress value was found in group II, followed by group IV, then by group III, and finally by group I. The fifth implant achieved the highest von Mises stress value in group II, followed by group IV, then by group I, and finally by group III. It was also noted that the von Mises stress generated by applying a 60-degree inclined force was the highest in group II for all implants. The lowest von Mises stress values were recorded in group III, except for implants 2 and 4 , whose lowest values were recorded in group I. 

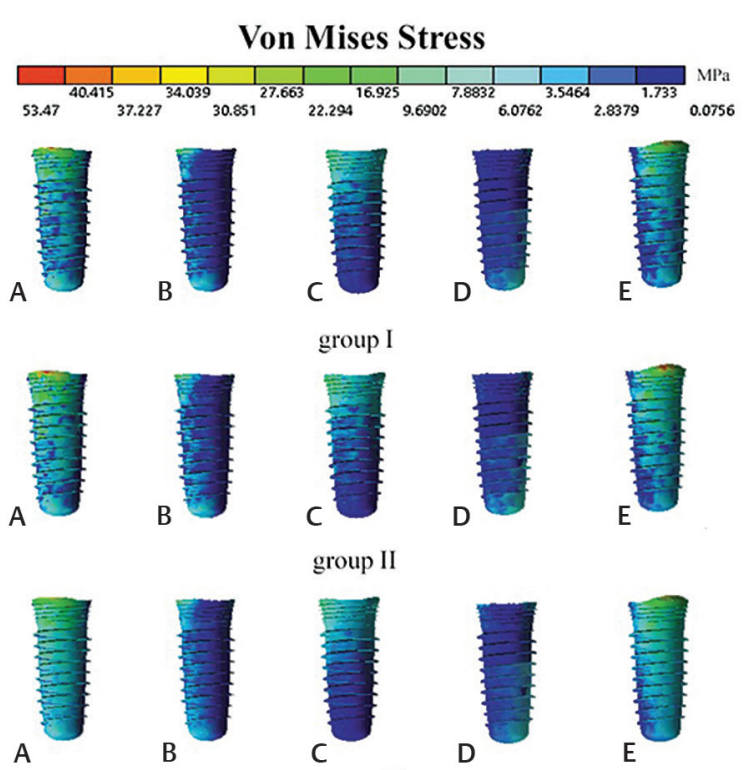

group III
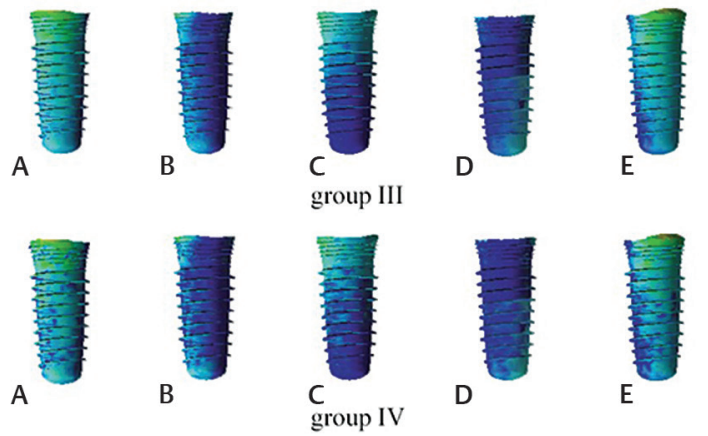

Fig. 6 von Mises stress distribution upon application of a vertical force on the implants of the studied groups.

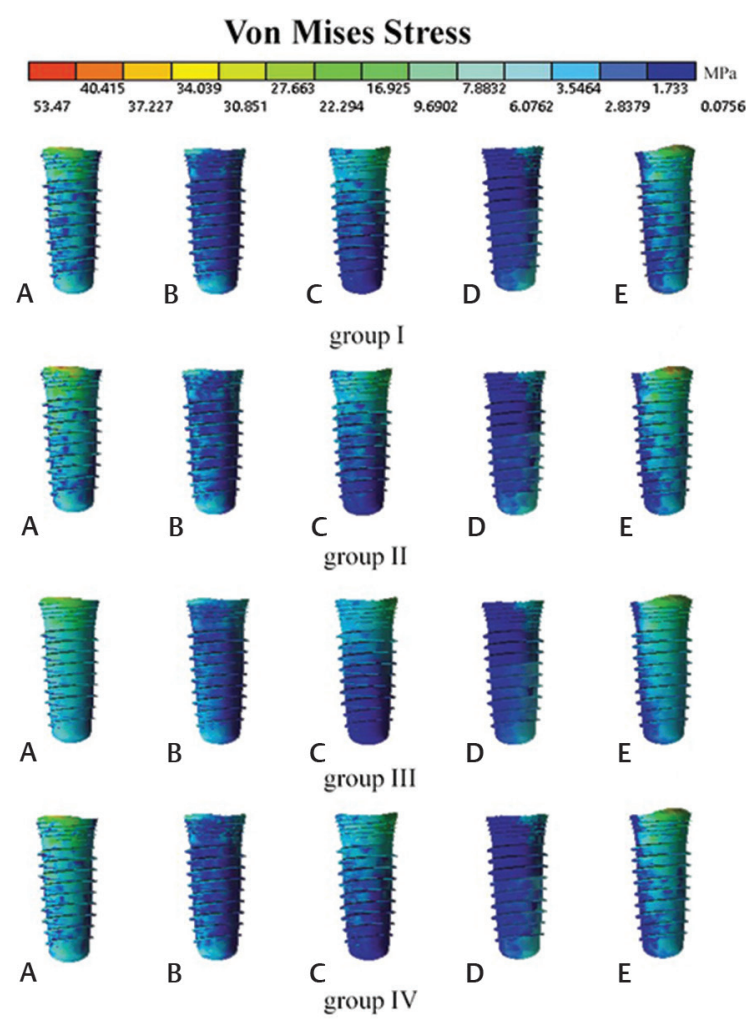

Fig. 7 von Mises stress distribution upon application of a 60-degree inclined force on the implants of the studied groups.

Table 3 von Mises strains at the bone-implant interfaces of the different studied groups upon application of vertical ( $V$ ) and inclined (I) forces (values multiplied by 1,000)

\begin{tabular}{|c|c|c|c|c|c|c|c|c|c|c|}
\hline & \multicolumn{2}{|c|}{ Implant 1} & \multicolumn{2}{|c|}{ Implant 2} & \multicolumn{2}{|c|}{ Implant 3} & \multicolumn{2}{|c|}{ Implant 4} & \multicolumn{2}{|c|}{ Implant 5} \\
\hline & $V$ & $I$ & $V$ & $I$ & $V$ & $I$ & $V$ & $I$ & $V$ & $I$ \\
\hline GI & 10.39 & 10.43 & 2.58 & 2.17 & 7.01 & 5.90 & 1.48 & 2.77 & 6.18 & 6.13 \\
\hline GII & 7.56 & 7.65 & 1.72 & 1.58 & 4.73 & 4.29 & 1.10 & 2.28 & 4.75 & 4.80 \\
\hline G III & 3.45 & 3.55 & 0.98 & 1.04 & 2.05 & 1.83 & 0.63 & 0.95 & 2.45 & 2.31 \\
\hline GIV & 5.67 & 5.73 & 1.56 & 1.47 & 3.78 & 4.15 & 0.95 & 1.81 & 4.10 & 3.89 \\
\hline
\end{tabular}

The values of the von Mises stress of the different groups were compared with those of group IV ( - Fig. 5B); implant 1 showed the greatest difference between groups, and implant 4 experienced a drop in its von Mises stress values, particularly for groups I and III. The generated color maps ( - Fig. 7) show that the highest stress concentration is found on implants 1,3 , and 5 , with a predominant concentration on the marginal bone and a few spots on the lingual side of implant 1 and the buccal side of implant 5 .

\section{von Mises Strain at the Bone-Implant Interface Upon Application of a Vertical Force}

The von Mises strain generated in the studied implants presented its highest value in group I, followed by group II, then group IV, and finally group III (-Table $\mathbf{3}$ ). The von Mises strain values of the different groups were compared with those of group IV ( $\boldsymbol{- \text { Fig. }} \mathbf{5 C}$ ); implant 3 showed the greatest difference among the groups, whereas implant 4 exhibited the smallest difference.
The von Mises strain color maps shown in - Fig. 8 indicate that the highest strain values are generated in the distal implants (implants 1 and 5), followed by the midsagittal implant (implant 3). High strain areas can be seen on the marginal bone of the bone-implant interface, particularly on the buccal side of the bone.

\section{von Mises Strain at the Bone-Implant Interface Upon Application of an Inclined Force}

Similar to the strains generated by the applied vertical forces, among all implants, group I showed the highest von Mises strain values, followed by group II, then group IV, and finally group III (-Table 3 ). In the comparison shown in - Fig. 5D, a significant difference is observed for implant 1. The color maps of generated von Mises strain shown in - Fig. $\mathbf{9}$ indicate that the highest strain values are located on implants 1 and 5 , followed by implant 3 . In addition, high strain areas can be clearly seen at the marginal bone of the bone-implant interface. 


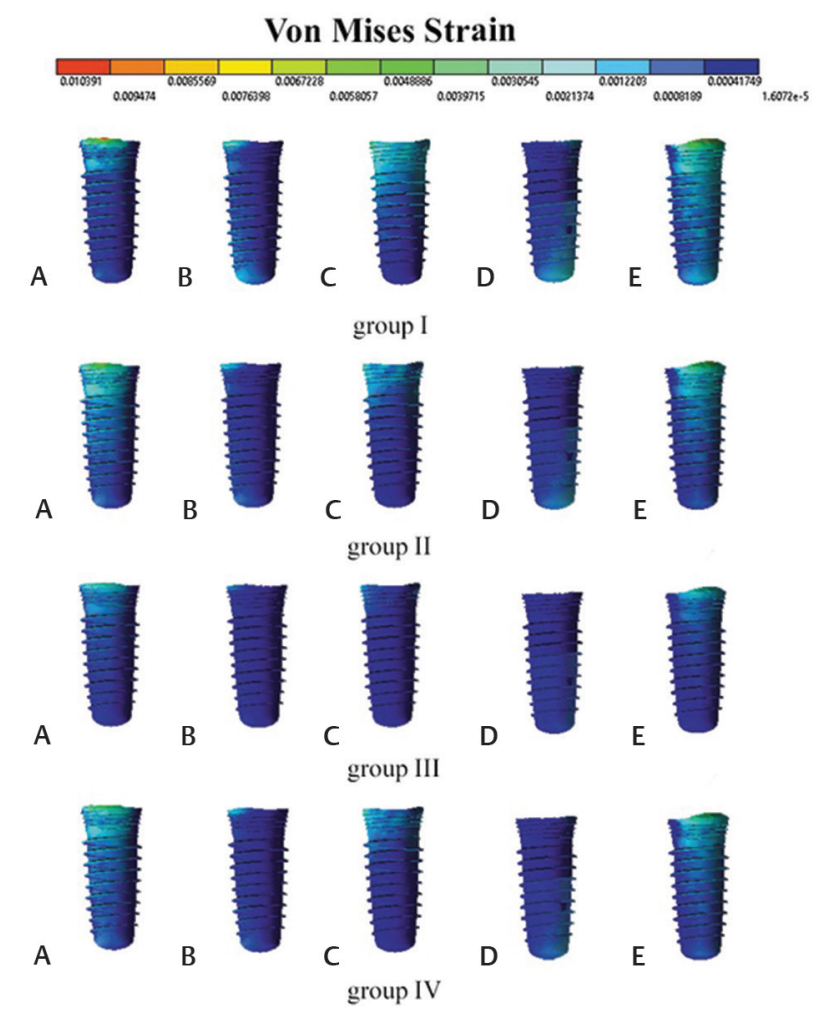

Fig. 8 von Mises strain distribution upon application of a vertical force on the studied implants.

\section{Discussion}

The assignment of heterogeneous properties to bone materials is a promising technique for performing patient-specific FEA in implant dentistry. Our study aimed to determine whether current calculation methods and correlation equations are accurate enough to provide reliable results, particularly in comparison with methods based on ordinary homogenous bone properties. Accordingly, three different methods for correlating bone grayscale level values with bone density and elastic modulus were used for material property assignment in the FEA mandibular bone model. Further, five implants were distributed along the mandibular bone to cover most of the expected implant areas. The boneimplant interface was the target of the analysis because this area presents the most significant effect on the treatment outcome.,17 In addition, both vertical and inclined forces were considered for loading the studied implants, based on literature values. ${ }^{23}$

The results of the study showed different bone responses to the applied load among groups, as seen in the resultant von Mises stress and strain values ( $\mathbf{- T a b l e s} \mathbf{2}$ and $\mathbf{3}$ ). By considering those stress values, group II showed the highest stresses among all the groups for all implants in both load conditions (i.e., vertical and inclined), except for the second implant during vertical force application. In group II, three different relations between bone density and elastic modulus were used; each of them was used within a certain density range. ${ }^{29,30}$ Moreover, the equations for the first and third

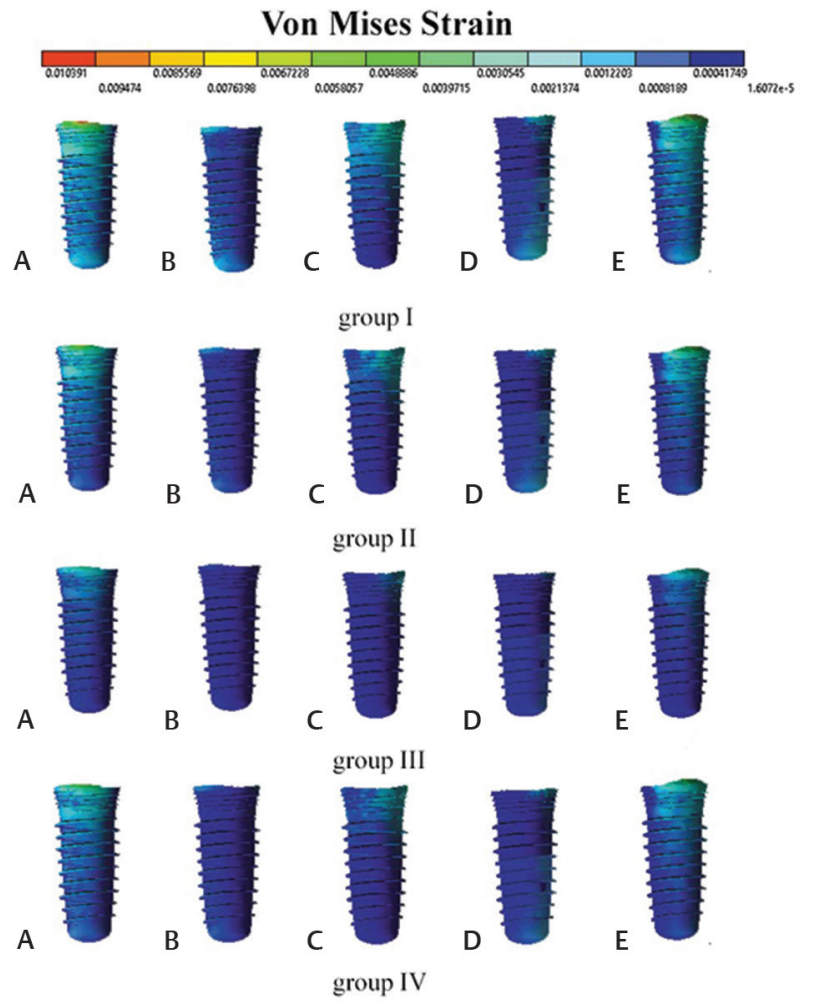

Fig. 9 von Mises strain distribution upon application of a 60-degree inclined force on the studied implants.

ranges depended on two power functions multiplied by a high-value constant. These calculations were represented by a wide elastic modulus range and a wide distribution among elements, as seen in the corresponding histogram of $\boldsymbol{\bullet}$ Fig. $\mathbf{3}$. Consequently, group II presented the most heterogeneous bone properties among all groups, with an abrupt change in the elastic modulus between layers of high and low density. These factors may enlarge the stress concentration region at the bone-implant interface, which extends from the surface to the inner layers of the bone within the length of the virtually placed implants. In contrast, group III presented an equation based on a linear function, which generated the lowest stress values.

Although the von Mises strain values for all implant showed the same intensity ranking results for all groups, the opposite was observed for the stress values, particularly when they were compared with the corresponding values of group IV (homogenous property group). This finding is in agreement with a previous study that compared the use of homogenous and heterogeneous properties in tibia bone; the authors of that work confirmed that the use of homogenous bone properties underestimated the resultant modal values. ${ }^{37}$ In addition, the stress values seem to be affected by the implant position. Furthermore, the two posterior implants and midsagittal implant exhibited higher stress values than implants 2 and 4 . These higher values could be attributed to their position in the dental arch, where they form the anterior and posterior boundaries of the arch, while implants 2 and 4 are protected in-between the other implants. These 
findings are in accordance with the results of other studies, which reported a higher stress on the posterior implants than the other implants of full-arch implant-supported prostheses. ${ }^{16,23,29}$

One of the outcomes of this study was the stress concentration in the marginal bone at the bone-implant interface; the results obtained match those reported in the literature. ${ }^{16,18,20,29,39}$ Marginal bone stress concentration was frequently seen in biomechanical studies in implant dentistry and could be associated by stress at the load application zones and the marginal edges of the pontic area. ${ }^{22}$ This finding was validated clinically by increased bone loss recorded in the crestal bone surrounding implant fixtures. ${ }^{39}$ The stress may increase on the opposite side of the force application zone when an inclined force is exerted, as found for implants 1 and 5 . This could be due to the lever-like action and moment generated when the inclined force is applied, as the fulcrum tends to be near the marginal area.

The findings of the present study highlight the lack of reliability of the results obtained when heterogeneous patient-specific bone properties based on current correlations between $\mathrm{HU}$, density, and elastic modulus are used. Consequently, this approach for bone property assignment should be used with caution to avoid misleading results. This may lead to overestimation or underestimation of the stress generated around certain implant as the study relied on using the heterogeneous material nature of the bone. Accordingly, more studies are required to ensure sound relations between the three parameters, GV, density, and elastic modulus of the human bones. Most of the current correlations are based on either the properties of a compact and cancellous long bone or default relations for general human bones. When the values were tested via a nanoindentation test, not all the mandibular bone zones could be verified using the suggested empirical expressions. ${ }^{24}$ Although the use of heterogeneous bone property assignment is a promising approach toward the validation of FEA, more in-depth studies are needed to determine the actual relations between the HU, density, and elastic modulus of mandibular bone.

The limitations of this study encompass various implant treatment designs and different bone densities as well as testing on more patients. More calculation methods, use of different contact types between the bone and implant surfaces, and the application of dynamic loads are recommended. Unless solid evidence is obtained about the accuracy of current correlations with respect to the actual bone properties in the mandible and maxilla, FEA results should be regarded as potentially misleading.

\section{Conclusion}

Within the limitations of the study, the different empirical expressions used herein for heterogeneous mechanical bone property assignment in FEA produced different stress and strain values at the bone-implant interface of the mandibular bone. Compared with the results of the homogenous bone property group, different results were obtained for the same implant positions when the heterogeneous mechanical bone property approach was used. The variations between their results raise doubts about their reliability as the results were influenced by implants' locations leading to misleading clinical simulations.

\section{Funding}

None.

\section{Conflict of Interest}

None declared.

\section{Acknowledgments}

The authors appreciate the technical support from Dr. Mohammed Salaheldin of PQI Arab Academy for science, Technology \& Maritime Transport.

\section{References}

1 Mao Q Su K, Zhou Y, Hossaini-Zadeh M, Lewis GS, Du J. Voxel-based micro-finite element analysis of dental implants in a human cadaveric mandible: tissue modulus assignment and sensitivity analyses. J Mech Behav Biomed Mater 2019;94:229-237

2 Ovesy M, Indermaur M, Zysset PK. Prediction of insertion torque and stiffness of a dental implant in bovine trabecular bone using explicit micro-finite element analysis. J Mech Behav Biomed Mater 2019;98:301-310

3 Chang JZ-C, Chen Y-J, Tung YY, et al. Effects of thread depth, taper shape, and taper length on the mechanical properties of mini-implants. Am J Orthod Dentofacial Orthop 2012;141(3):279-288

4 Cha JY, Pereira MD, Smith AA, et al. Multiscale analyses of the bone-implant interface. J Dent Res 2015;94(3):482-490

5 Hou X, Weiler MA, Winger JN, Morris JR, Borke JL. Rat model for studying tissue changes induced by the mechanical environment surrounding loaded titanium implants. Int J Oral Maxillofac Implants 2009;24(5):800-807

6 Heckmann SM, Karl M, Wichmann MG, Winter W, Graef F, Taylor TD. Loading of bone surrounding implants through three-unit fixed partial denture fixation: a finite-element analysis based on in vitro and in vivo strain measurements. Clin Oral Implants Res 2006;17(3):345-350

7 Sotto-Maior BS, Mercuri EGF, Senna PM, Assis NMSP, Francischone CE, Del Bel Cury AA. Evaluation of bone remodeling around single dental implants of different lengths: a mechanobiological numerical simulation and validation using clinical data. Comput Methods Biomech Biomed Engin 2016;19(7):699-706

8 Alrbata RH, Yu W, Kyung HM. Biomechanical effectiveness of cortical bone thickness on orthodontic microimplant stability: an evaluation based on the load share between cortical and cancellous bone. Am J Orthod Dentofacial Orthop 2014;146(2):175-182

9 Zarone F, Apicella A, Nicolais L, Aversa R, Sorrentino R. Mandibular flexure and stress build-up in mandibular full-arch fixed prostheses supported by osseointegrated implants. Clin Oral Implants Res 2003;14(1):103-114

10 Bulaqi HA, Mousavi Mashhadi M, Geramipanah F, Safari H, Paknejad M. Effect of the coefficient of friction and tightening speed on the preload induced at the dental implant complex with the finite element method. J Prosthet Dent 2015;113(5):405-411

11 Choi KS, Park SH, Lee JH, Jeon YC, Yun MJ, Jeong CM. Stress distribution on scalloped implants with different microthread and connection configurations using three-dimensional finite element analysis. Int J Oral Maxillofac Implants 2012;27(3):e29-e38 
12 Lin D, Li Q Li W, Duckmanton N, Swain M. Mandibular bone remodeling induced by dental implant. J Biomech 2010;43(2):287-293

13 Chang Y, Tambe AA, Maeda Y, Wada M, Gonda T. Finite element analysis of dental implants with validation: to what extent can we expect the model to predict biological phenomena? A literature review and proposal for classification of a validation process. Int J Implant Dent 2018;4(1):7

14 Bujtár P, Sándor GKB, Bojtos A, Szűcs A, Barabás J. Finite element analysis of the human mandible at 3 different stages of life. Oral Surg Oral Med Oral Pathol Oral Radiol Endod 2010;110(3):301-309

15 Merdji A, Bouiadjra BB, Chikh BO, et al. Stress distribution in dental prosthesis under an occlusal combined dynamic loading. Mater Des 2012;36:705-713

16 Hussein MO, Rabie ME. Three-dimensional nonlinear contact finite element analysis of mandibular All-on-4 design. J Oral Implantol 2015;41(2):e12-e18

17 Guven S, Beydemir K, Dundar S, Eratilla V. Evaluation of stress distributions in peri-implant and periodontal bone tissues in 3- and 5-unit tooth and implant-supported fixed zirconia restorations by finite elements analysis. Eur J Dent 2015;9(3):329-339

18 Bonnet AS, Postaire M, Lipinski P. Biomechanical study of mandible bone supporting a four-implant retained bridge: finite element analysis of the influence of bone anisotropy and foodstuff position. Med Eng Phys 2009;31(7):806-815

19 Chevalier Y, Santos I, Müller PE, Pietschmann MF. Bone density and anisotropy affect periprosthetic cement and bone stresses after anatomical glenoid replacement: a micro finite element analysis. J Biomech 2016;49(9):1724-1733

20 Al-Zordk W, Ghazy M, El-Anwar M. Stress analysis around reduced-diameter zirconia and titanium one-piece implants with and without microthreads in the neck: experimental and finite element analysis. Int J Oral Maxillofac Implants 2020;35(2):305-312

21 Lencioni KA, Noritomi PY, Macedo AP, Ribeiro RF, Pereira AR. Influence of different implants on the biomechanical behavior of a tooth-implant fixed partial dentures: a three-dimensional finite element analysis. J Oral Implantol 2020;46(1):27-34

22 Eraslan O, Inan O, Secilmis A. The effect of framework design on stress distribution in implant-supported FPDs: a 3-D FEM study. Eur J Dent 2010;4(4):374-382

23 Dogru SC, Cansiz E, Arslan YZ. A review of finite element applications in oral and maxillofacial biomechanics. J Mech Med Biol 2018;18(02):1830002

24 Xin P, Nie P, Jiang B, Deng S, Hu G, Shen SGF. Material assignment in finite element modeling: heterogeneous properties of the mandibular bone. J Craniofac Surg 2013;24(2):405-410

25 Rho J-Y, Hobatho MC, Ashman RB. Relations of mechanical properties to density and CT numbers in human bone. Med Eng Phys 1995;17(5):347-355
26 Lettry S, Seedhom BB, Berry E, Cuppone M. Quality assessment of the cortical bone of the human mandible. Bone 2003;32(1):35-44

27 Keyak JH, Lee IY, Skinner HB. Correlations between orthogonal mechanical properties and density of trabecular bone: use of different densitometric measures. J Biomed Mater Res 1994;28(11):1329-1336

28 Keller TS. Predicting the compressive mechanical behavior of bone. J Biomech 1994;27(9):1159-1168

29 Horita S, Sugiura T, Yamamoto K, Murakami K, Imai Y, Kirita T. Biomechanical analysis of immediately loaded implants according to the "All-on-Four" concept. J Prosthodont Res 2017;61(2):123-132

30 Keyak JH, Rossi SA, Jones KA, Skinner HB. Prediction of femoral fracture load using automated finite element modeling. J Biomech 1998;31(2):125-133

31 Cheng KJ, Liu YF, Wang JH, et al. Biomechanical behavior of mandibles reconstructed with fibular grafts at different vertical positions using finite element method. J Plast Reconstr Aesthet Surg 2019;72(2):281-289

32 Nutu E, Ahmad S, Pastrama S. Influence of bone elastic properties on the predicted stress distribution in the dental implant vicinity. Mater Today Proc 2017;4(5):5904-5908

33 Liu YF, Wang R, Baur DA, Jiang XF. A finite element analysis of the stress distribution to the mandible from impact forces with various orientations of third molars. J Zhejiang Univ Sci B 2018;19(1):38-48

34 Shu J-H, Yao J, Zhang Y-L, Chong DYR, Liu Z. The influence of bilateral sagittal split ramus osteotomy on the stress distributions in the temporomandibular joints of the patients with facial asymmetry under symmetric occlusions. Medicine (Baltimore) 2018;97(25):e11204

35 Marcián P, Florian Z, Horáčková L, Kaiser J, Borák L. Microstructural finite-element analysis of influence of bone density and histomorphometric parameters on mechanical behavior of mandibular cancellous bone structure. Diffus Defect Data Solid State Data Pt B Solid State Phenom 2016;258:362-365

$36 \mathrm{Hu} \mathrm{Z}$, Petoukhov SV, He M, Advances in Artificial Systems for Medicine and Education II. vol 902. Cham, Switzerland: Springer International Publishing; 2020

37 Yassine RA, Elham MK, Mustapha S, Hamade RF. Heterogeneous versus homogeneous material considerations in determining the modal frequencies of long tibia bones. J Eng Sci Med Diagn Ther 2018;1(2)

38 Turkyilmaz I, Ozan O, Yilmaz B, Ersoy AE. Determination of bone quality of 372 implant recipient sites using Hounsfield unit from computerized tomography: a clinical study. Clin Implant Dent Relat Res 2008;10(4):238-244

39 Caetano GM, Pauletto P, Mezzomo LA, Rivaldo EG. Crestal bone changes in different implants designs: a prospective clinical trial. Eur J Dent 2019;13(4):497-502 